\title{
Sudden Cardiac Death in Three Generations of the Same Family: A case report.
}

\author{
M.M. Zahurul Alam Khan ${ }^{1}$, Abdul Wadud Chowdhury², Tunaggina Afrin Khan ${ }^{3}$, Md. Gaffar Amin², \\ Md. Nur Alam ${ }^{4}$, Khandaker Md. Nurus Sabah², Mohammed Abaye Deen Saleh ${ }^{2}$ \\ ${ }^{1}$ Department of Cardiology, BIRDEM General Hospital \& Ibrahim Medical College, ${ }^{2}$ Department of \\ Cardiology, Dhaka Medical College Hospital, ${ }^{3}$ Department of Cardiology, Anwer Khan Modern Medical \\ College \& Hospital, ${ }^{4}$ Department of Cardiology, National Institute of Cardiovascular Diseases.
}

Key Words : Hypertrophic Cardiomyopathy, Sudden Cardiac Death.

\begin{abstract}
:
Every one of us has heard about tragic and sudden death of a healthy young person and which is often stated as 'inexplicable'. The current case report focuses on a 20 year old young man with hypertrophic cardiomyopathy facing premature death with history of similar sudden premature death of his grandmother, father and brother. Hypertrophic cardiomyopathy is the commonest cause of sudden cardiac death in young adults and is also an important substrate for heart failure disability at any age.
\end{abstract}

(Cardiovasc. j. 2015; 8(1): 73-77)

\section{Introduction:}

Hypertrophic Cardiomyopathy (HCM) is a disease that involves the myocardium but it is not the result of hypertension or any valvular, congenital, arterial or pericardial abnormalities. ${ }^{1}$ Various imaging modalities and electrophysiological study have enhanced our knowledge regarding the systolic and diastolic abnormalities in HCM. Moreover the results of molecular genetic studies have resulted in a quantum leap. ${ }^{2}$ Majority of the patients with HCM have at least one other affected first degree relative. It affects males more than females. ${ }^{3}$ Mortality rate is approximately $1 \%$ per year. $^{4}$

\section{Case report:}

Our patient a 20 year old young man, normotensive, nondiabetic, nonsmoker \& nonalcoholic, was admitted in the Coronary Care Unit of Dhaka Medical College Hospital in December, 2012 with the complaints of nonproductive cough and progressively increasing shortness of breath associated with bilateral leg swelling for 15 days. At first his breathlessness occurred in moderate to severe exertion, and it was gradually increasing day by day. It was associated with orthopnoea and paroxysmal nocturnal dyspnoea (PND) and made him bedbound for 7- 8 days prior to admission. At the same time, he also noticed abdominal swelling and facial puffiness. He gave history of occasional leg swelling for few months before admission which could be alleviated by taking some medications. Those periods of leg swelling were associated with palpitation and used to last for days to weeks. According to his mother's statement he never played like an average kid during his early childhood and used to retire early while playing. However, he used to perform his daily activities without any trouble. She first noticed his problem when he was unable to walk to nearby school at the age of 7 to 8 years. At that time, he used to experience palpitation, shortness of breath, retrosternal chest discomfort and he needed to take rest for a while before reaching school. Gradually, his problems began to increase and he quit going to school. His elder brother died suddenly at the age of 12 years without any known preceding illness, and so did his father and his grandmother before reaching 40 years of age.

On admission he was dyspnoic (breathing 24 times per minute). Pulse was very feeble, blood pressure was not recordable. JVP was raised to $14 \mathrm{~cm} \mathrm{H}_{2} \mathrm{O}$ with a prominent a wave. Apex was shifted to anterior axillary line at left $5^{\text {th }}$ intercostal space with double apical impulse and it was forceful, sustained. First heart sound was soft, and

Address of Correspondence: Dr. M. M. Zahurul Alam Khan, Department of Cardiology, BIRDEM General Hospital \& Ibrahim Medical College, Dhaka, Bangladesh. Email: zaksaifsk@yahoo.com 
pulmonary component of the second heart sound was loud. A rough pansystolic murmur of grade 3/ 6 was heard at the apical area with radiation towards axilla and back. An ejection systolic murmur in left lower parasternal area was heard which increased during expiration and squatting. Bilateral basal fine crepitations in both lung fields were found. He had tender hepatomegaly $(9 \mathrm{~cm})$. Other systemic examinations were normal. His ECG showed atrial flutter with variable block with heart rate of $70 \mathrm{bpm}(\mathrm{Fig} 1)$;

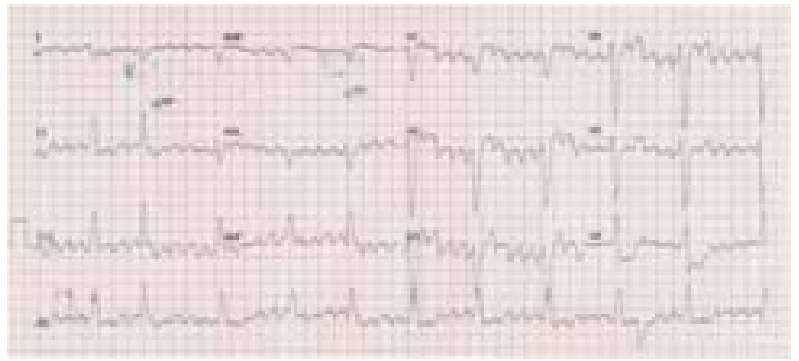

Fig 1: ECG at admission showing atrial flutter with variable block

After excluding the presence of any intracardiac thrombus, he was converted to sinus rhythm with immediate DC cardioversion using 50 Joules (Fig 2).

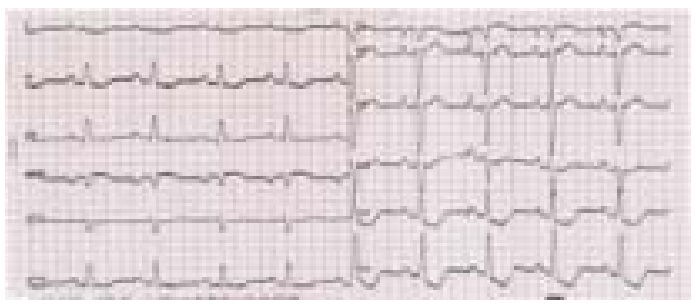

Fig.-2: ECG after DC cardioversion

After DC cardioversion his pulse rate was approximately $60 \mathrm{bpm}$ and blood pressure was 95/ $60 \mathrm{~mm} \mathrm{Hg}$. Chest roentgenogram (Fig.3) revealed cardiomegaly.

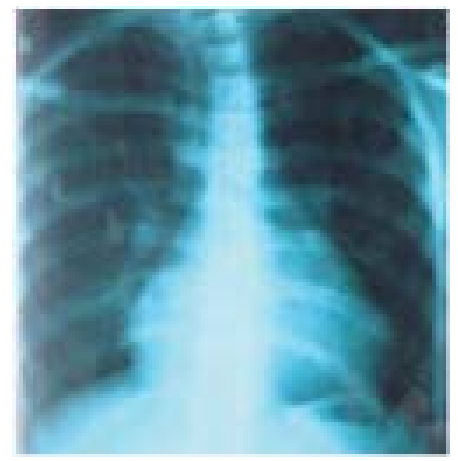

Fig.-3: CXR PA view showing cardiomegaly
Echocardiography revealed asymmetrical septal hypertrophy (IVSd: LVPWd= $1.8 \mathrm{~cm}: 1.1 \mathrm{~cm}$, Fig. 4) with restrictive pattern of diastolic dysfunction; prolapse of anterior mitral leaflet during ventricular systole (Fig. 5), mitral regurgitation(MR Grade II); left atrium, right ventricle \& right atrium were dilated. Pulmonary artery systolic pressure was $43 \mathrm{mmHg}$.

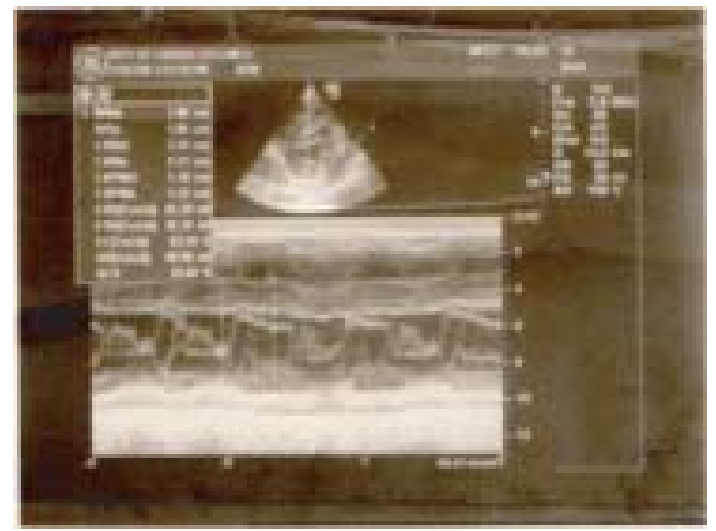

Fig.-4: IVS:LVPW $=1.8 \mathrm{~cm}: 1.1 \mathrm{~cm}$

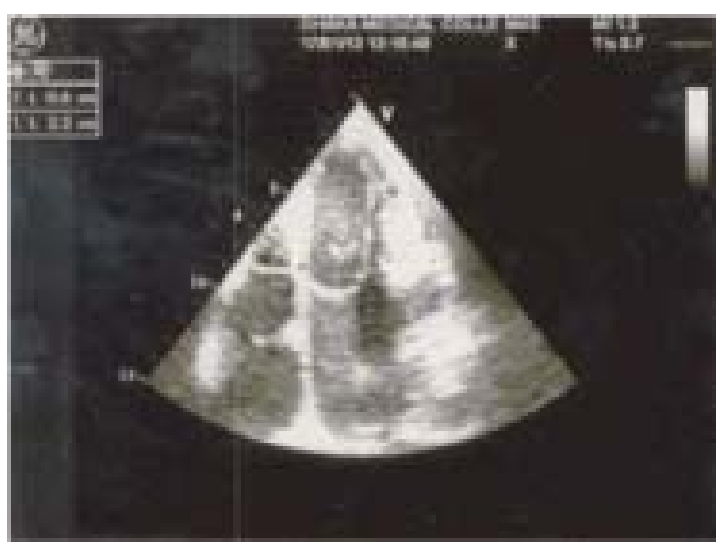

Fig.-5: Prolapse of AML towards LA $6 \mathrm{~mm}$ from annular margin

His baseline investigations were unremarkable except raised ALT $255 \mathrm{U} / \mathrm{L}$. He underwent coronary angiogram and it revealed normal epicardial coronary arteries (Fig.6) with right dominant right coronary artery (RCA, Fig. 7). However, there was myocardial bridging with systolic luminal obliteration of left anterior descending artery $(\mathrm{LAD})$ and diagonal $\left(\mathrm{D}_{1}\right)$ with subsequent diastolic refilling(Fig 8,9). 


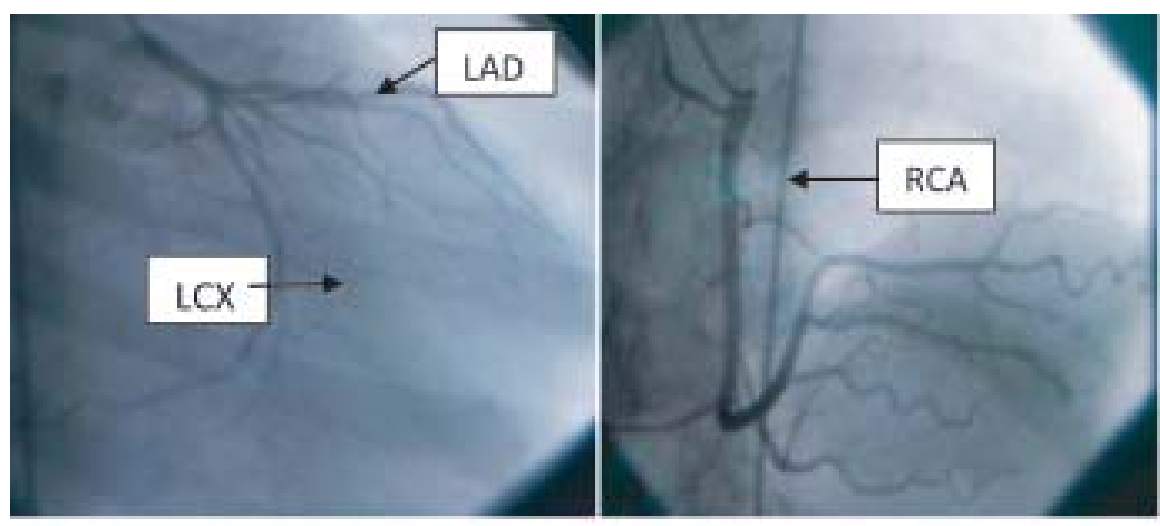

Fig.-6 \& 7: Normal epicardial coronary arteries with superdominant right supply.
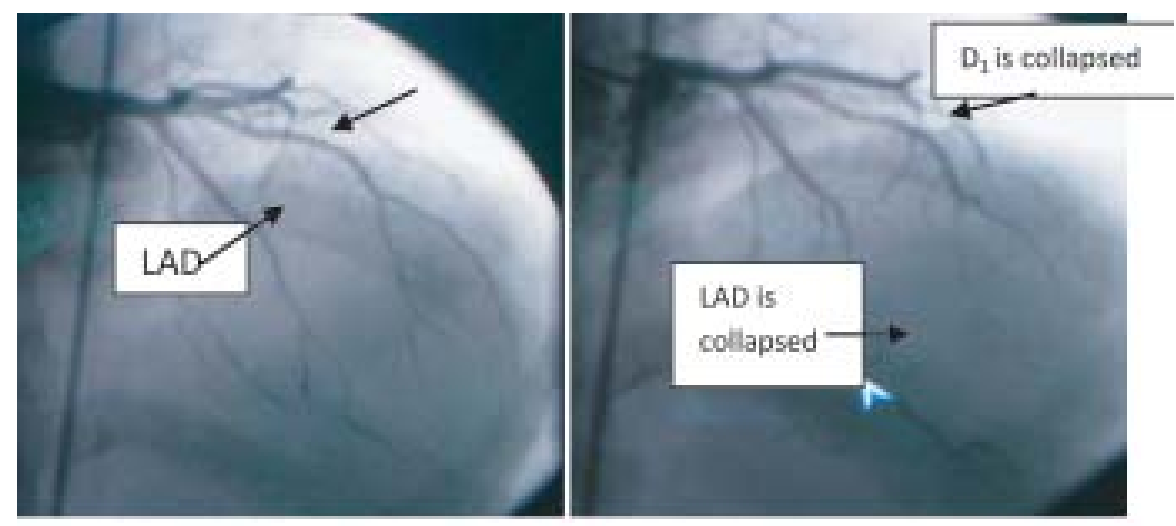

Fig.-8 \& 9: Myocardial bridging in LAD and its branches with systolic collapse of coronary arteries.

Screening of his family members revealed asymmetrical septal hypertrophy in his younger sister but she was hemodynamically alright and clinically symptomless.

Our diagnosis was congestive cardiac failure and atrial flutter with variable block (reverted to sinus) due to hypertrophic cardiomyopathy with mitral valve prolapse with MR (Grade-II). He was prescribed frusemide, warfarin, spironolactone, propranolol, and amiodarone. Patient's family was informed about the necessity of implanting automated implantable cardioverter defibrillator (AICD), but his family could not afford it. One week after the patient was discharged in a haemodynamically stable condition, his brother informed us that he has had the same miserable destiny just like his brother, father \& grandmother i.e. sudden cardiac death.

\section{Discussion:}

HCM is characterized by "unexplained LV hypertrophy associated with nondilated LV chamber in the absence of another cardiac or systemic disease (i.e. aortic valve stenosis, systemic hypertension) with or without obstruction to LV outflow tract." ${ }^{5}$ It is the most common form of cardiomyopathies which is inherited as autosomal dominant pattern. ${ }^{5}$ This is consistent with the pattern of inheritance in our case. HCM is usually recognized by $L V$ wall thickness $\geq 15 \mathrm{~mm}$ and diagnosis is more presumable if associated with family history of HCM, cardiac symptoms, and ECG finding. ${ }^{5}$ Common sites of hypertrophy are septum, LV apex, and LV posterior wall with or without septal involvement. Our case had asymmetrical septal hypertrophy. This occurs with mutation of genes involving beta myosin heavy chain, myosin binding protein $\mathrm{C}$ etc. ${ }^{6}$ 
Gross anatomy shows asymmetrical septal hypertrophy, small LV cavity, fibrosis of endocardium, dilated left atrium, and left ventricle outflow tract obstruction by systolic anterior motion of mitral valve. ${ }^{7}$ In this case, these were all found except any evidence of LV outflow tract obstruction. Histologically, there is myocardial disarray, and reduced size of septal intramural coronary arteries, ${ }^{7}$ which is partly responsible for exercise intolerance. Myocardial ischemia occurs due to supply-demand mismatch \& small narrow lumen intramural coronary arteries. Myocardial bridging is frequent in HCM (in this case in LAD and $\mathrm{D}_{1}$ ) and contributes to increased risk of sudden cardiac death. ${ }^{2,5}$ Diastolic dysfunction is obvious due to high systolic contraction with non-uniform relaxation; resultant increased pulmonary arterial pressure lead to dyspnoea which were also present in this case. ${ }^{2,5}$

Most of the patients are asymptomatic with incidental diagnosis of HCM. Symptomatic ones come with angina, dyspnoea, syncope, palpitation. ${ }^{2,5}$ One of the most important complication is sudden cardiac death (SCD), which may occur either as the first presentation of HCM or may occur at any time in symptomatic patient. Cardiac arrest occurs by asystole, pulseless ventricular tachycardia (VT), ventricular fibrillation (VF), and electromechanical dissociation (EMD). ${ }^{2,5}$ It occurs more in young asymptomatic or less symptomatic patients. ${ }^{2}$ Though our patient was symptomatic but strong family history and history of arrhythmia put him in danger zone for SCD.

In precordium double or triple apical impulse, third or fourth heart sounds may be present. An ejection systolic crescendo-decrescendo murmur in left lower parasternal border is heard with radiation to heart base and apex, but not to carotid. Intensity of murmur can be augmented by exercise, and leg raising. $^{2}$

ECG shows left ventricular hypertrophy, arrhythmia, pre-excitation, Q wave and ST-T changes. Echocardiography is the gold standard test and left ventricular outflow tract (LVOT) gradient is $>30 \mathrm{mmHg}$ in obstructive variety. In this case, LVOT gradient was normal. Cardiac MRI is superior to echocardiography to provide accurate diagnosis, distribution and extent of HCM. If IHD is suspected then coronary angiogram, MPI, PET,
SPECT may be done. ${ }^{2,5}$

Shirley et al. ${ }^{8}$ found out that $40-50 \%$ of SCD in young patients occurs due to HCM, more with anomalous origin of coronary arteries. Corrado et al. ${ }^{9}$ suggested on important congenital causes of SCD in patients less than 35 years old which are mitral-valve prolapse, HCM, and myocardial bridging. Dermengiu et al. ${ }^{10}$ reported a case of SCD in a 20 year old athlete without any previous illness due to HCM having both anomalous origin of RCA and myocardial bridging in LAD \& LCX at autopsy. Spirito et al. ${ }^{11}$ and Alonso et al. ${ }^{12}$ reported SCD occuring in patients with HCM with wall thickness $>30 \mathrm{~mm}$. Our case is unique in presentation in the following way. He had history of sudden cardiac death in his family but septal wall thickness was $<30 \mathrm{~mm}$, having associated mitral valve prolapse, and myocardial bridging involving $\mathrm{LAD}$ and $\mathrm{D}_{1}$ but no anomalous coronary artery origin. He also had documented atrial flutter with variable block but not VT or VF.

General measures should be taken in all patients of $\mathrm{HCM}$ i.e. low to moderate level of exercise, no competitive sport, screening of all $1^{\text {st }}$ degree relatives by echocardiography and DNA analysis, and avoidance of dehydration, high dose diuretics, vasodilators. For medical treatment, â blockers, verapamil \& diltiazem are the first line drug therapy as they reduce heart rate and force of contraction, improve diastolic relaxation with minimal effect on afterload. These drugs improve exercise tolerance and angina but have no effect on preventing SCD. Target HR is $60 \mathrm{bpm}$. CCB is contraindicated in severe LVOT gradient. Dihydropyridines are avoided. Disopyramide can be added as additional drug. If $\mathrm{AF}$ is present with $\mathrm{MR}$, anticoagulation is recommended. In case of acute hypotension in HOCM, fluid loading is done, if it fails IV phenylephrine is used. Positive ionotropic drugs are contraindicated. In asymptomatic patients, no treatment is necessary. Only optimization of obesity, BP, DM, hyperlipidemia are all that is needed. For diastolic dysfunction, beta blocker or CCB is started, if not controlled diuretics are added. ARB, ACEI, Dihydropyridines, statins may be used in nonobstructive variety. In intractable diastolic dysfunction cardiac transplantation is an option..$^{5,13}$ Septal myectomy or ablation are done for 
obstructive variety of HCM with LVOT gradient $>50 \mathrm{~mm} \mathrm{Hg} \&$ in drug refractory cases. These procedures have mortality benefit. Pacemaker implantation (DDD) is indicated in patients with bradycardia or who will need ICD. But result is suboptimal. So it is rarely used now. AICD is indicated in high risk patients of SCD and has proven mortality benefit. ${ }^{14}$ Our patient could not afford it because of financial constrain.

\section{Conclusion:}

$\mathrm{HCM}$ is the commonest cause of sudden cardiac death in young individuals. Particularly vulnerable ones are with the positive family history of premature death. 5 yearly screening of all first degree relatives are recommended. AICD is the treatment of choice in patients of $\mathrm{HCM}$ with risk of SCD.

\section{Conflict of Interest - None.}

\section{References:}

1. Maron BJ, McKenna WJ, Danielson GK, Kappenberger LJ, Kuhn HJ, Seidman CE et al. American Collegeof Cardiology/European Society of Cardiology Clinical Expert Consensus Document on Hypertrophic Cardiomyopathy. A report of the American College of Cardiology Task Force on Clinical Expert Consensus Documents and the European Society of Cardiology Committee for Practice Guidelines Committee to Develop an Expert Consensus Document on Hypertrophic Cardiomyopathy. J Am Coll Cardiol 2003;42:1687-1713.

2. Wigle ED, Rakowski H, Kimball BP. Hypertrophic Cardiomyopathy, Clinical Spectrum and Treatment. Circulation 1995; 92: 1680-1692.

3. S Agarwal, C Chetan, S Iliyas. Sudden death - a case of hypertrophic cardiomyopathy. JIAFM 2004; 26(4): 157 159.

4. Daralammouri Y, El Garhy M, Same K, Lauer B. Hypertrophic Cardiomyopathy mimicking Acute Anterior Myocardial Infarction Associated with Sudden Cardiac Death. Case Reports in Medicine, Volume 2012, Article ID 236154.
5. Gersh BJ, Maron BJ, Bonow RO, Dearani JA, Fifer MA, Link MS et al. ACCF/AHA Guideline for the Diagnosis and Treatment of Hypertrophic Cardiomyopathy: A Report of the American College of Cardiology Foundation/American Heart Association Task Force on Practice Guidelines. Circulation 2011; 124: e783-e831.

6. Maron BJ, Towbin JA, Taha G. Contemporary Definitions and Classification of the Cardiomyopathies. An American Heart Association Scientific Statement From the Council on Clinical Cardiology, Heart Failure and Transplantation Committee; Quality of Care and Outcomes Research and Functional Genomics and Translational Biology Interdisciplinary Working Groups; and Council on Epidemiology and Prevention. Circulation 2006; 113: 1807-1816.

7. Noureldin RA, Liu S, Nacif MS. The diagnosis of hypertrophic cardiomyopathy by cardiovascular magnetic resonance. $J$ Cardiov Magn Reson 2012;14:17. s

8. Shirley KW, Adirim TA. Sudden Cardiac Death in Young Athletes. Clin Ped Emerg Med 2005;6:194-199.

9. Corrado D, Basso C, Schiavon M, Thiene G. Screening for Hypertrophic Cardiomyopathy in Young Athletes. N Engl J Med 1998; 339:364-369.

10. Dermengiu D, Ceausu M, Rusu MC, Dermengiu S, Curc GC, Hostiuc S. Sudden death associated with borderline Hypertrophic Cardiomyopathy and multiple coronary anomalies. Case report and literature review. Rom J Leg Med 2010; 1: 3-12.

11. Spirito P, Bellone P, Harris KM, Bernabò P, Bruzzi P, Maron BJ. Magnitude of left ventricular hyperthophy and risk of sudden death in hypertrophic cardiomyopathy. N Engl J Med 2000;342:1778-1785.

12. Pedrote A, Morales FJ, Garcý 'a-Riesco L, Errazquin F. Documented exercise-induced cardiac arrest in apaediatric patient with hypertrophic cardiomyopathy. Europace 2006; 8: 430-433.

13. Fifer MA, Vlahakes GJ. Contemporary Reviews in Cardiovascular Medicine. Management of Symptoms in Hypertrophic Cardiomyopathy. Circulation 2008; 117: 429-439.

14. Maron BJ, Spirito P, Shen WK, Haas TS, Formisano F, Link MS et al. Implantable cardioverterdefibrillators and prevention of sudden cardiac death in hypertrophic cardiomyopathy. JAMA. 2007;298:405-412. 